\title{
Genetic Variability Estimates for Yield and Yield Components Traits and Quality Traits in Rice (Oryza sativa L.)
}

Hari Ram Kumar Bandi ${ }^{*}$, P.V. Satyanarayana
, D. Ratna Babu
N. Chamundeswari

${ }^{1}$ Department of Genetics and Plant Breeding, Agricultural College, Bapatla, A.P. - 522 101, India

${ }^{2}$ Plant Breeding, APRRI \& RARS, Maruteru, W.G, A.P.-534 122, India

${ }^{3}$ Genetics and Plant Breeding, Advanced P.G Studies, Lam, Guntur, A.P.-522 034, India

${ }^{4}$ Agricultural College, Bapatla, A.P.-522 101, India

${ }^{5}$ APRRI \& RARS, Maruteru, W.G, A.P.-534 122, India

*Corresponding author

\section{A B S T R A C T}

An experiment with a total of 36 rice entries consisted of 28 cross and eight parental lines were evaluated to estimate the variability parameters for 10 yield attributing character, 6

\section{Keywords}

Variability,

Heritability,

Genetic advance,

Rice

\section{Article Info}

Accepted:

07 April 2018

Available Online:

10 May 2018 physical quality traits; 8 cooking and chemical quality traits of rice cultures. Analysis of variance revealed the existence of significant differences among cultures for all traits studied. High PCV coupled with GCV was recorded for gel consistency and alkali spreading value revealed wider variability for these traits. Moderate PCV and GCV was observed for total number of tillers per plant, number of grains per panicle, LAI at maximum tillering stage, volume expansion ratio, water uptake, amylose content and grain yield per plant indicating the moderate variability. High heritability coupled with high genetic advance as per cent of means for total number of tillers per plant, number of grains per panicle, leaf area index at maximum tillering stage, water uptake, gel consistency, alkali spreading value, amylose content and grain yield per plant, revealed the role of additive gene effect and simple selection procedures may be effective for improving these traits. High heritability coupled with moderate genetic advance as per cent of mean was observed for days to $50 \%$ flowering, days to maturity, plant height, test weight, kernel breadth after cooking and volume expansion ratio, revealed that role of both additive and non-additive gene effects in the inheritance of these traits.

\section{Introduction}

Rice (Oryza sativa L., $2 \mathrm{n}=24$ ) is the most important food crop in the world (Asia, Latin America, parts of Africa and the Middle East), which accounts for more than $21 \%$ of the calorific needs of the world's population and up to $76 \%$ of the calorific intake of the population of South East Asia (Melissa et al., 2009). About $90 \%$ of the world's rice is grown and consumed in Asia. According to the projections made by the Population Foundation of India, the country's population will be 1546 million by the end of 2030 and 
1824 million by the end of 2050. It is estimated that the demand for rice will be 121.2 million tonnes by the year 2030 and 137.3 million tonnes by the year 2050 (CRRI VISION 2050).

Due to various socio-economic constraints, a chance of bringing more area under rice cultivation is very remote. Hence to achieve the target of increased rice production, it requires raising the production per unit area. Emphasis is being diverted towards the maximum utilization of land by producing more rice yield per unit area per unit time for which there is a need to improve different traits related to grain yield.

Yield is the resultant product of various morphological, physiological and biological components. Yield can be enhanced using the various cultivars with wider genetic variability in hybridization programme. The presence of adequate genetic variability is regarded as the fundamental pre-requisite to launch any crop improvement programme and the success depends on its magnitude in a given species. The genotypic coefficient of variation measures the magnitude of genetic variability. Since, it reflects the heritable portion of variability.

Information on heritability along with genetic advance will be helpful in prediction of genetic gain possible in improvement of a character by selection. Heritability and genetic advance are regarded as important selection parameters. Burton (1952) suggested that genetic variation along with heritability estimates would give a better idea about the efficiency of selection. Hence, wider range of genetic variability helps in selecting desired genotypes. In addition to the genetic variability, knowledge on heritability and genetic advance helps the breeder to employ the suitable breeding strategy. Therefore, it is necessary to have knowledge of genetic variability, heritability and genetic advance present in the available genetic material.

\section{Materials and Methods}

Twenty eight $F_{1} s$ along with eight parents were sown in rabi, 2016-15 at Andhra Pradesh Rice Research Institute (APRRI) and Regional Agricultural Research Station (RARS), Maruteru, West Godavari, Andhra Pradesh in a Randomized Complete Block Design with three replications. Observations were recorded on ten randomly chosen plants for twenty four various yield attributing traits and quality characters, i.e.10 yield attributing characters viz., days to $50 \%$ flowering, days to maturity, plant height $(\mathrm{cm})$, number of tillers per plant, number of ear bearing tillers per plant, panicle length $(\mathrm{cm})$, number of grains per panicle, test weight $(\mathrm{g})$, leaf area index at maximum tillering stage and grain yield per plant $(\mathrm{g}) ; 6$ physical quality traits such as hulling recovery per cent, milling recovery per cent, head rice recovery, kernel length $(\mathrm{mm})$, kernel breadth (mm), kernel L/B ratio; 8 cooking and chemical quality traits such as kernel length after cooking (mm), kernel breadth after cooking (mm), kernel elongation ratio, volume expansion ratio, water uptake value $(\mathrm{ml})$, gel consistency $(\mathrm{mm})$, alkali digestion value and amylose content (\%). The data were subjected to statistical analysis and various genetic parameters such as PCV, GCV, heritability and genetic advance were worked out as per Johnson et al., (1955) and Hanson (1963).

\section{Results and Discussion}

The mean sum of squares due to genotypes was highly significant at $1 \%$ level of significance for all the characters studied in the present investigation. This indicated the existence of considerable variability for all characters studied among the genotypes. Hence, it offers a better scope for further improvement of breeding material by the 
selection of promising genotypes in rice breeding programme. The mean sum of squares of various characters was presented in Table 2.

The parameters of genetic variability such as mean, range, phenotypic coefficient of variation (PCV), genotypic coefficient of variation (GCV), heritability and genetic advance as percentage of mean (GA) were estimated for all the quantitative characters are presented in Table 1 and 3.

The variation for yield attributing traits viz., days to $50 \%$ flowering ranged from 68.67 $($ BPT $3291 \times$ MCM 100) to 108.00 (MTU $1064 \times$ MTU 7029) with a mean of 94.34 , days to maturity from 97.67 days (BPT $3291 \times$ MCM 100) to 138.00 days (MTU $1064 \times$ MTU 7029) with a mean of 123.69 days, plant height ranged from $92.90 \mathrm{~cm}$ (BPT $3291 \times$ MTU 7029) to $130.18 \mathrm{~cm}(\mathrm{MCM} 100 \times$ MTU 7029 ) with a mean of $114.90 \mathrm{~cm}$, total number of tillers per plant varied from 10.33 (MTU $1121)$ to 17.67 (MTU $1001 \times$ MTU 1140) with a mean of 14.87 .

The variation for number of ear bearing tillers per plant ranged from $8.67(\mathrm{MCM} 100 \times \mathrm{MTU}$ 7029 ) to 14.00 (BPT 3291) with a mean of 11.85. Panicle length per plant in the material studied varied from 24.47 (BPT 3291) to 31.20 (MTU $1140 \times$ MTU 1064) with a mean of 28.73. Number of grains per panicle was from 141.23 (BPT 3291) to 270.89 (MTU $1140 \times$ MTU 1064) with a mean of 213.78 .

The range for test weight was from 18.20 (MTU 7029) to 24.33 (MTU $1001 \times$ MCM 100 ) with a mean of 21.15 . Leaf area index at maximum tillering stage was from 2.09 (BPT $3291 \times$ MTU 1121) to 3.72 (MTU $1001 \times$ MTU 1140) with a mean of 2.70 . Grain yield per plant ranged from $20.21 \mathrm{~g}$ (MTU $1001 \times$ MTU 1061) to $38.03 \mathrm{~g}$ (BPT-329) with a mean of $28.75 \mathrm{~g}$.
The physical quality traits viz., hulling recovery per cent was ranged from $77.82 \%$ $($ MTU $1061 \times$ MTU 7029) to $82.24 \%$ (BPT $3291 \times$ MTU 1064) with a mean of $79.85 \%$. Head rice recovery per cent ranged from $62.67 \%$ (MTU 7029) to $74.98 \%$ (MTU $1001 \times$ MTU 1140) with a mean of $62.09 \%$. Kernel length $(\mathrm{mm})$ ranged from $5.31 \mathrm{~mm}$ (MTU 7029) to $6.06 \mathrm{~mm}($ BPT $3291 \times$ MTU 1140) with a mean of $5.67 \mathrm{~mm}$.

Kernel breadth $(\mathrm{mm})$ varied from $1.93 \mathrm{~mm}$ (MTU $1001 \times$ MTU 1064) to $2.46 \mathrm{~mm}$ (MTU $1001 \times$ MTU 7029) with a mean of $2.23 \mathrm{~mm}$. Kernel length/breadth ratio noted for this trait varied from 2.37 (MTU $1001 \times$ MTU 7029) to 2.90 (MTU $1001 \times$ MTU 1064) with a mean of 2.54 .

The variation for cooking and cooking quality characters such as kernel length after cooking (mm) ranged from 8.73 (MTU 7029) to 10.09 (MTU 1061) with a mean of 9.53. Kernel breadth after cooking varied from 2.56 (MTU $1001 \times$ MTU 1064) to 3.25 (MTU $1001 \times$ MTU 1140) with a mean of 2.89. Kernel elongation ratio ranged from 1.55 (MCM 100 $\times$ MTU 7029) to $1.77($ MTU $1001 \times$ MTU 1121 ) with a mean of 1.68. Volume expansion ratio ranged from $3.79($ BPT $3291 \times$ MTU 1140) to $5.82($ MTU $1001 \times$ MTU 7029) with a mean of 4.73 .

Water uptake $(\mathrm{ml})$ varied from 121.67 (BPT $3291 \times$ MTU-100) to 256.67 (MTU 1001) with a mean of 183.29. Gel consistency ranged from 41.66 (MTU $1064 \times$ MTU 1061) to 98.27 (MTU $1140 \times$ MTU 1061) with a mean of 67.69 . The range of variation noted for alkali spreading value varied from 2.50 $(\mathrm{MCM} 100 \times \mathrm{MTU} 1121)$ to $6.17(\mathrm{MTU}$ 1061) with a mean of 4.30. Amylose content possessed significant variation which was ranged from $18.86(\mathrm{MCM} 100 \times$ MTU 1061) to 32.53 (MTU $1121 \times$ MTU 7029) with a mean of 23.70 . 
Table.1 Mean performance of 8 parents and $28 \mathrm{~F}_{1} \mathrm{~s}$ in rice (Oryza sativa L.)

\begin{tabular}{|c|c|c|c|c|c|c|c|c|c|c|c|c|}
\hline S. No. & Parents and $F_{1} S$ & DFF & DM & PH & TNTP & TEBTP & PL & NGPP & TW & LAI & GYPP & HP \\
\hline 1 & MTU 1001 & 89.33 & 119.67 & 113.05 & 11.33 & 9.33 & 26.51 & 160.56 & 23.42 & 2.43 & 23.04 & 78.35 \\
\hline 2 & BPT 3291 & 95.33 & 125.33 & 106.44 & 10.67 & 8.67 & 24.47 & 141.23 & 19.43 & 2.17 & 20.21 & 79.08 \\
\hline 3 & MCM 100 & 100.33 & 129.67 & 105.02 & 12.00 & 10.33 & 26.63 & 195.13 & 18.43 & 2.49 & 20.39 & 80.38 \\
\hline 4 & MTU 1121 & 92.00 & 122.00 & 106.39 & 10.33 & 9.67 & 26.57 & 181.76 & 19.70 & 3.23 & 22.27 & 78.69 \\
\hline 5 & MTU 1140 & 105.67 & 134.67 & 115.67 & 12.33 & 10.33 & 26.41 & 225.50 & 21.03 & 3.28 & 22.98 & 80.14 \\
\hline 6 & MTU 1064 & 104.67 & 132.67 & 113.06 & 12.00 & 10.67 & 26.64 & 232.50 & 19.56 & 2.35 & 23.32 & 79.97 \\
\hline 7 & MTU 1061 & 107.00 & 136.67 & 114.84 & 12.33 & 11.00 & 26.97 & 214.77 & 19.85 & 3.02 & 23.02 & 79.02 \\
\hline 8 & MTU 7029 & 105.67 & 135.00 & 109.84 & 13.00 & 10.67 & 25.03 & 171.96 & 18.20 & 2.43 & 23.12 & 78.79 \\
\hline 9 & MTU $1001 \times$ BPT 3291 & 83.67 & 114.00 & 109.66 & 14.67 & 12.00 & 29.20 & 211.09 & 23.42 & 3.47 & 24.79 & 81.26 \\
\hline 10 & MTU $1001 \times$ MCM 100 & 95.67 & 126.67 & 117.43 & 15.67 & 11.67 & 29.71 & 213.49 & 24.33 & 2.86 & 35.20 & 80.20 \\
\hline 11 & MTU $1001 \times$ MTU 1121 & 83.00 & 113.00 & 113.11 & 14.33 & 12.33 & 29.01 & 193.77 & 23.06 & 2.47 & 34.72 & 79.47 \\
\hline 12 & MTU $1001 \times$ MTU 1140 & 90.67 & 121.00 & 125.40 & 17.67 & 12.67 & 29.27 & 214.69 & 22.46 & 3.72 & 36.55 & 80.67 \\
\hline 13 & MTU $1001 \times$ MTU 1064 & 92.67 & 122.67 & 116.23 & 15.33 & 12.67 & 28.87 & 208.05 & 21.44 & 2.78 & 36.14 & 79.72 \\
\hline 14 & MTU $1001 \times$ MTU 1061 & 82.33 & 111.33 & 118.46 & 16.67 & 13.33 & 28.58 & 222.60 & 21.87 & 2.50 & 38.03 & 79.60 \\
\hline 15 & MTU $1001 \times$ MTU 7029 & 96.67 & 124.33 & 96.00 & 14.00 & 12.00 & 29.54 & 191.86 & 20.52 & 2.13 & 25.98 & 79.11 \\
\hline 16 & BPT $3291 \times$ MCM 100 & 68.67 & 97.67 & 113.10 & 16.00 & 13.00 & 30.59 & 225.98 & 23.19 & 2.54 & 26.27 & 80.45 \\
\hline 17 & BPT $3291 \times$ MTU 1121 & 95.67 & 123.33 & 108.53 & 16.00 & 13.33 & 29.52 & 211.90 & 19.78 & 2.09 & 26.92 & 78.98 \\
\hline 18 & BPT $3291 \times$ MTU 1140 & 96.67 & 125.00 & 129.83 & 16.00 & 13.33 & 31.11 & 230.48 & 23.36 & 3.52 & 34.30 & 81.67 \\
\hline 19 & BPT $3291 \times$ MTU 1064 & 86.00 & 117.00 & 103.90 & 16.00 & 12.67 & 29.59 & 216.85 & 19.85 & 2.45 & 29.11 & 82.24 \\
\hline 20 & BPT $3291 \times$ MTU 1061 & 92.67 & 120.67 & 112.53 & 17.33 & 12.00 & 29.33 & 228.24 & 20.92 & 2.79 & 33.59 & 79.40 \\
\hline 21 & BPT $3291 \times$ MTU 7029 & 92.67 & 120.00 & 92.90 & 15.00 & 10.67 & 28.97 & 212.60 & 19.35 & 2.17 & 26.71 & 79.36 \\
\hline 22 & MCM $100 \times$ MTU 1121 & 87.67 & 118.00 & 116.98 & 16.67 & 12.00 & 30.36 & 205.97 & 21.62 & 2.36 & 25.47 & 78.67 \\
\hline 23 & MCM $100 \times$ MTU 1140 & 98.67 & 128.33 & 123.12 & 17.33 & 13.00 & 28.28 & 257.28 & 21.73 & 3.62 & 35.31 & 79.73 \\
\hline 24 & MCM $100 \times$ MTU 1064 & 82.67 & 111.00 & 122.29 & 16.00 & 12.33 & 28.94 & 215.40 & 20.06 & 2.56 & 35.93 & 81.12 \\
\hline 25 & MCM $100 \times$ MTU 1061 & 92.00 & 122.00 & 115.11 & 16.00 & 10.67 & 30.65 & 248.88 & 19.98 & 2.45 & 32.76 & 79.52 \\
\hline 26 & MCM $100 \times$ MTU 7029 & 91.33 & 118.33 & 130.18 & 17.33 & 14.00 & 29.61 & 225.94 & 21.37 & 2.11 & 29.29 & 80.68 \\
\hline 27 & MTU $1121 \times$ MTU 1140 & 91.33 & 120.67 & 123.36 & 14.67 & 12.00 & 29.14 & 227.67 & 21.96 & 3.07 & 36.18 & 80.67 \\
\hline 28 & MTU $1121 \times$ MTU 1064 & 96.67 & 128.00 & 107.31 & 14.33 & 12.33 & 29.06 & 186.82 & 22.64 & 2.57 & 24.40 & 78.97 \\
\hline 29 & MTU $1121 \times$ MTU 1061 & 90.67 & 120.33 & 113.61 & 16.00 & 12.33 & 29.47 & 185.59 & 22.14 & 2.63 & 25.78 & 80.22 \\
\hline 30 & MTU $1121 \times$ MTU 7029 & 98.33 & 127.33 & 112.09 & 14.67 & 13.00 & 28.65 & 190.59 & 20.00 & 2.16 & 23.75 & 80.98 \\
\hline 31 & MTU $1140 \times$ MTU 1064 & 96.00 & 126.33 & 123.02 & 15.67 & 13.67 & 31.20 & 270.89 & 22.85 & 3.17 & 26.55 & 79.19 \\
\hline 32 & MTU $1140 \times$ MTU 1061 & 99.33 & 128.33 & 129.10 & 15.67 & 12.67 & 29.58 & 249.50 & 23.33 & 3.04 & 27.85 & 79.65 \\
\hline 33 & MTU $1140 \times$ MTU 7029 & 102.00 & 129.00 & 121.27 & 16.00 & 12.00 & 27.97 & 214.84 & 20.14 & 3.12 & 32.34 & 79.54 \\
\hline 34 & MTU $1064 \times$ MTU 1061 & 100.33 & 129.33 & 121.44 & 17.33 & 12.00 & 29.09 & 236.17 & 19.88 & 2.49 & 35.68 & 80.15 \\
\hline 35 & MTU $1064 \times$ MTU 7029 & 108.00 & 138.00 & 116.70 & 14.67 & 11.33 & 29.66 & 205.34 & 19.76 & 2.34 & 28.24 & 81.14 \\
\hline \multirow[t]{5}{*}{36} & MTU $1061 \times$ MTU 7029 & 104.67 & 135.67 & 119.28 & 14.33 & 11.00 & 30.20 & 270.11 & 20.71 & 2.49 & 28.91 & 77.82 \\
\hline & Overall Mean & 94.35 & 123.69 & 114.90 & 14.87 & 11.85 & 28.73 & 213.78 & 21.15 & 2.70 & 79.85 & 72.65 \\
\hline & C.V. & 2.04 & 1.84 & 1.77 & 6.49 & 8.64 & 4.41 & 5.28 & 2.05 & 4.68 & 1.43 & 1.66 \\
\hline & S.Em & 1.11 & 1.32 & 1.17 & 0.56 & 0.59 & 0.73 & 6.51 & 0.25 & 0.07 & 0.66 & 0.70 \\
\hline & C.D. $5 \%$ & 3.14 & 3.71 & 3.31 & 1.57 & 1.67 & 2.06 & 18.37 & 0.71 & 0.21 & 1.86 & 1.96 \\
\hline
\end{tabular}

DFF: Days to 50\% Flowering; DM: Days to Maturity; PH: Plant Height; TNTP: Total Number of Tillers per Plant; TEBTP: Total number of Ear Bearing Tillers per Plant; PL: Panicle Length per plant; NGPP: Number of Grains per Panicle; TW: Test Weight; LAI: Leaf Area Index at maximum tillering stage. 
Table.1 Mean performance of 8 parents and $28 \mathrm{~F}_{1} \mathrm{~s}$ in rice (Oryza sativa L.)

\begin{tabular}{|c|c|c|c|c|c|c|c|c|c|c|c|c|c|c|}
\hline S. No. & Parents and $\mathrm{F}_{1} \mathrm{~S}$ & MP & HRR & KL & KB & $\mathrm{L} / \mathrm{B}$ & KLAC & KBAC & KLER & VER & WU & GC & ASV & $\mathrm{AC}$ \\
\hline 1 & MTU 1001 & 67.87 & 63.91 & 5.93 & 2.31 & 2.57 & 10.05 & 2.81 & 1.69 & 4.91 & 256.67 & 52.27 & 5.50 & 25.45 \\
\hline 2 & BPT 3291 & 69.50 & 64.12 & 5.43 & 2.03 & 2.67 & 9.28 & 2.59 & 1.71 & 5.15 & 165.00 & 53.34 & 3.67 & 24.53 \\
\hline 3 & MCM 100 & 71.56 & 66.55 & 5.56 & 2.22 & 2.50 & 9.06 & 2.71 & 1.63 & 5.11 & 150.00 & 57.95 & 3.39 & 20.23 \\
\hline 4 & MTU 1121 & 69.50 & 64.52 & 5.78 & 2.09 & 2.76 & 9.40 & 2.58 & 1.63 & 4.80 & 171.67 & 57.27 & 2.94 & 22.21 \\
\hline 5 & MTU 1140 & 70.52 & 66.00 & 5.69 & 2.24 & 2.54 & 10.03 & 2.66 & 1.76 & 4.67 & 225.00 & 49.04 & 4.06 & 25.08 \\
\hline 6 & MTU 1064 & 72.36 & 65.80 & 5.66 & 2.11 & 2.69 & 9.98 & 2.61 & 1.76 & 4.47 & 156.67 & 62.88 & 6.11 & 24.22 \\
\hline 7 & MTU 1061 & 71.55 & 66.98 & 5.78 & 2.04 & 2.83 & 10.09 & 2.77 & 1.75 & 4.66 & 208.33 & 62.86 & 6.17 & 21.05 \\
\hline 8 & MTU 7029 & 67.20 & 62.67 & 5.31 & 2.13 & 2.49 & 8.73 & 3.07 & 1.64 & 3.92 & 185.00 & 46.12 & 3.11 & 22.48 \\
\hline 9 & MTU $1001 \times$ BPT 3291 & 74.19 & 69.82 & 5.85 & 2.31 & 2.54 & 9.56 & 2.83 & 1.64 & 4.41 & 151.67 & 45.97 & 5.17 & 21.68 \\
\hline 10 & MTU $1001 \times$ MCM 100 & 73.98 & 69.18 & 5.53 & 2.18 & 2.53 & 9.55 & 2.80 & 1.73 & 4.24 & 153.33 & 69.23 & 5.17 & 22.78 \\
\hline 11 & MTU $1001 \times$ MTU 1121 & 73.13 & 70.23 & 5.65 & 2.23 & 2.54 & 10.00 & 2.72 & 1.77 & 4.05 & 165.00 & 86.70 & 4.28 & 23.89 \\
\hline 12 & MTU $1001 \times$ MTU 1140 & 77.92 & 74.98 & 5.61 & 2.32 & 2.42 & 9.78 & 2.99 & 1.74 & 4.21 & 190.00 & 94.08 & 5.06 & 26.51 \\
\hline 13 & MTU $1001 \times$ MTU 1064 & 73.99 & 67.73 & 5.60 & 1.93 & 2.90 & 9.77 & 2.56 & 1.75 & 4.44 & 255.00 & 76.36 & 4.94 & 22.70 \\
\hline 14 & MTU 1001 × MTU 1061 & 74.89 & 66.04 & 5.84 & 2.36 & 2.47 & 9.85 & 2.84 & 1.69 & 5.80 & 211.67 & 87.36 & 5.72 & 21.56 \\
\hline 15 & MTU $1001 \times$ MTU 7029 & 72.83 & 65.42 & 5.84 & 2.46 & 2.37 & 9.81 & 3.08 & 1.68 & 5.82 & 170.00 & 50.75 & 4.28 & 20.86 \\
\hline 16 & BPT $3291 \times$ MCM 100 & 74.10 & 70.46 & 5.57 & 2.27 & 2.46 & 9.38 & 3.10 & 1.68 & 4.62 & 121.67 & 91.37 & 2.56 & 28.73 \\
\hline 17 & BPT $3291 \times$ MTU 1121 & 71.45 & 64.96 & 5.77 & 2.34 & 2.47 & 9.97 & 3.09 & 1.73 & 4.09 & 185.00 & 44.47 & 3.33 & 23.50 \\
\hline 18 & BPT $3291 \times$ MTU 1140 & 76.51 & 70.54 & 6.06 & 2.25 & 2.69 & 9.55 & 3.20 & 1.58 & 3.79 & 166.67 & 94.10 & 2.78 & 23.61 \\
\hline 19 & BPT $3291 \times$ MTU 1064 & 76.24 & 68.97 & 5.71 & 2.34 & 2.44 & 9.88 & 3.06 & 1.73 & 4.74 & 165.00 & 78.37 & 5.17 & 19.84 \\
\hline 20 & BPT $3291 \times$ MTU 1061 & 70.69 & 63.60 & 5.51 & 2.16 & 2.55 & 9.05 & 3.23 & 1.64 & 4.71 & 185.00 & 43.04 & 4.67 & 31.04 \\
\hline 21 & BPT $3291 \times$ MTU 7029 & 74.05 & 64.63 & 5.64 & 2.23 & 2.53 & 9.56 & 3.11 & 1.70 & 4.78 & 185.00 & 97.96 & 5.89 & 22.64 \\
\hline 22 & MCM $100 \times$ MTU 1121 & 70.88 & 64.13 & 5.74 & 2.23 & 2.58 & 9.15 & 2.99 & 1.59 & 4.78 & 190.00 & 70.41 & 2.50 & 30.80 \\
\hline 23 & MCM $100 \times$ MTU 1140 & 73.79 & 70.69 & 5.36 & 2.15 & 2.49 & 9.06 & 3.25 & 1.69 & 4.53 & 193.33 & 56.57 & 2.61 & 20.16 \\
\hline 24 & MCM $100 \times$ MTU 1064 & 72.51 & 68.60 & 5.76 & 2.26 & 2.55 & 9.50 & 3.05 & 1.65 & 5.44 & 193.33 & 42.03 & 5.22 & 22.40 \\
\hline 25 & MCM $100 \times$ MTU 1061 & 73.58 & 67.61 & 5.65 & 2.26 & 2.50 & 9.87 & 3.22 & 1.75 & 4.76 & 163.33 & 97.92 & 3.44 & 18.86 \\
\hline 26 & MCM $100 \times$ MTU 7029 & 73.87 & 69.45 & 5.88 & 2.38 & 2.47 & 9.08 & 2.86 & 1.55 & 5.05 & 190.00 & 92.18 & 2.61 & 21.75 \\
\hline 27 & MTU $1121 \times$ MTU 1140 & 73.39 & 71.51 & 5.49 & 2.15 & 2.55 & 9.54 & 2.79 & 1.74 & 4.40 & 163.33 & 87.11 & 3.56 & 21.94 \\
\hline 28 & MTU $1121 \times$ MTU 1064 & 70.14 & 65.07 & 5.57 & 2.25 & 2.48 & 9.43 & 2.77 & 1.69 & 5.41 & 190.00 & 45.14 & 5.28 & 25.39 \\
\hline 29 & MTU $1121 \times$ MTU 1061 & 73.00 & 67.33 & 5.61 & 2.27 & 2.47 & 9.09 & 2.56 & 1.62 & 4.70 & 200.00 & 55.87 & 4.78 & 22.67 \\
\hline 30 & MTU $1121 \times$ MTU 7029 & 72.97 & 63.22 & 5.71 & 2.25 & 2.54 & 9.34 & 3.03 & 1.63 & 5.48 & 181.67 & 88.72 & 5.00 & 32.53 \\
\hline 31 & MTU $1140 \times$ MTU 1064 & 71.96 & 68.54 & 5.73 & 2.27 & 2.53 & 9.62 & 2.76 & 1.68 & 4.91 & 163.33 & 55.14 & 2.56 & 21.17 \\
\hline 32 & MTU $1140 \times$ MTU 1061 & 75.37 & 70.60 & 5.60 & 2.30 & 2.43 & 9.43 & 2.56 & 1.69 & 4.69 & 171.67 & 98.27 & 4.56 & 25.36 \\
\hline 33 & MTU $1140 \times$ MTU 7029 & 70.44 & 67.38 & 5.67 & 2.27 & 2.50 & 9.17 & 2.75 & 1.62 & 4.06 & 163.33 & 87.85 & 4.44 & 23.26 \\
\hline 34 & MTU $1064 \times$ MTU 1061 & 73.56 & 65.11 & 5.75 & 2.25 & 2.55 & 9.87 & 2.73 & 1.72 & 4.81 & 223.33 & 41.66 & 2.61 & 20.99 \\
\hline 35 & MTU $1064 \times$ MTU 7029 & 74.62 & 65.32 & 5.55 & 2.22 & 2.51 & 9.47 & 3.10 & 1.71 & 4.79 & 205.00 & 48.03 & 5.67 & 29.36 \\
\hline \multirow[t]{5}{*}{36} & MTU $1061 \times$ MTU 7029 & 71.28 & 63.48 & 5.73 & 2.30 & 2.50 & 9.11 & 3.08 & 1.59 & 5.10 & 183.33 & 68.56 & 5.94 & 21.95 \\
\hline & Overall Mean & 67.09 & 5.67 & 2.23 & 2.54 & 9.53 & 2.89 & 1.68 & 4.73 & 183.29 & 67.69 & 4.30 & 23.70 & 28.75 \\
\hline & C.V. & 2.01 & 1.46 & 1.59 & 1.69 & 1.74 & 3.80 & 2.27 & 3.27 & 3.78 & 4.18 & 4.11 & 2.92 & 5.55 \\
\hline & S.Em & 0.78 & 0.05 & 0.02 & 0.02 & 0.10 & 0.06 & 0.02 & 0.09 & 4.00 & 1.63 & 0.10 & 0.40 & 0.92 \\
\hline & C.D. $5 \%$ & 2.20 & 0.13 & 0.06 & 0.07 & 0.27 & 0.18 & 0.06 & 0.25 & 11.29 & 4.60 & 0.29 & 1.13 & 2.60 \\
\hline
\end{tabular}

HP\%: Hulling percentage; MP\%: Milling percentage; HRR\%: Head Rice Recovery; KL: Kernel Length; KB: Kernel Breadth ;L/B: Kernel L/B ratio; KLAC:

Kernel Length After Cooking; KBAC: Kernel Breadth After Cooking; KLER: Kernel Linear Elongation Ratio; VER: Volume Expansion Ratio; WU: Water Uptake; GC: Gel Consistency; ASV: Alkali Spreading Value; AC: Amylose Content. 
Table. 2 Analysis of variance for grain yield, yield component characters and quality trait of $28 \mathrm{~F}_{1} \mathrm{~S}$ and 8 parents in rice (Oryza sativa L.)

\begin{tabular}{|c|c|c|c|c|c|c|c|c|c|c|c|c|c|}
\hline $\begin{array}{l}\text { Source of } \\
\text { variations }\end{array}$ & d.f. & $\begin{array}{c}\text { Days to } \\
50 \% \\
\text { flowering }\end{array}$ & $\begin{array}{l}\text { Days to } \\
\text { maturity }\end{array}$ & $\begin{array}{l}\text { Plant } \\
\text { height } \\
(\mathrm{cm})\end{array}$ & $\begin{array}{c}\text { Total } \\
\text { number } \\
\text { of tillers } \\
\text { per plant }\end{array}$ & $\begin{array}{c}\text { Number } \\
\text { of ear } \\
\text { tillers } \\
\text { per } \\
\text { plant }\end{array}$ & $\begin{array}{l}\text { Panicle } \\
\text { length } \\
\text { per plant } \\
\text { (cm) }\end{array}$ & $\begin{array}{l}\text { Number of } \\
\text { grains per } \\
\text { panicle }\end{array}$ & $\begin{array}{c}\text { Test } \\
\text { weight } \\
\text { (gm) }\end{array}$ & $\begin{array}{l}\text { Leaf area } \\
\text { index at } \\
\text { maximum } \\
\text { tillering } \\
\text { stage }\end{array}$ & $\begin{array}{c}\text { Hulling } \\
\text { percentage }\end{array}$ & $\begin{array}{c}\text { Milling } \\
\text { percentage }\end{array}$ & $\begin{array}{l}\text { Head rice } \\
\text { recovery }\end{array}$ \\
\hline \multicolumn{14}{|c|}{ Mean sum of squares } \\
\hline Replications & 2 & 1.037 & 1.750 & 0.073 & 0.065 & 0.009 & 0.124 & 4.896 & 0.039 & 0.006 & 0.045 & 0.053 & 0.001 \\
\hline Treatments & 35 & $207.085 * *$ & $207.036 * *$ & $218.032 * *$ & $11.681 * *$ & $4.751 * *$ & $7.762 * *$ & $2333.950 * *$ & $7.616 * *$ & $0.636^{* *}$ & $16.181 * *$ & $24.776 * *$ & $0.072 * *$ \\
\hline Error & 70 & 3.723 & 5.188 & 4.127 & 0.931 & 1.047 & 1.605 & 127.268 & 0.188 & 0.016 & 1.451 & 1.824 & 0.007 \\
\hline Total & 107 & 211.844 & 213.974 & 222.231 & 12.678 & 5.808 & 9.491 & 2466.114 & 7.843 & 0.657 & 17.677 & 26.653 & 0.080 \\
\hline
\end{tabular}

Table. 2 Analysis of variance for grain yield, yield component characters and quality trait of $28 \mathrm{~F}_{1} \mathrm{~S}$ and 8 parents in rice (Oryza sativa L.)

\begin{tabular}{|c|c|c|c|c|c|c|c|c|c|c|c|c|c|}
\hline $\begin{array}{l}\text { Source of } \\
\text { variations }\end{array}$ & d.f. & $\begin{array}{l}\text { Kernel } \\
\text { length } \\
(\mathrm{mm})\end{array}$ & $\begin{array}{l}\text { Kernel } \\
\text { breadth } \\
(\mathrm{mm})\end{array}$ & $\begin{array}{c}\text { Kernel } \\
\text { L/B ratio }\end{array}$ & $\begin{array}{l}\text { Kernel } \\
\text { length } \\
\text { after } \\
\text { cooking } \\
(\mathrm{mm})\end{array}$ & $\begin{array}{c}\text { Kernel } \\
\text { breadth } \\
\text { after } \\
\text { cooking } \\
(\mathrm{mm})\end{array}$ & $\begin{array}{c}\text { Kernel } \\
\text { elongatio } \\
\mathbf{n} \text { ratio }\end{array}$ & $\begin{array}{l}\text { Volume } \\
\text { expansion } \\
\text { ratio }\end{array}$ & $\begin{array}{l}\text { Water } \\
\text { uptake } \\
\text { (ml) }\end{array}$ & $\begin{array}{c}\text { Gel } \\
\text { consistency } \\
(\mathbf{m m})\end{array}$ & $\begin{array}{c}\text { Alkali } \\
\text { spreading } \\
\text { value }(\mathbf{m m})\end{array}$ & $\begin{array}{c}\text { Amylose } \\
\text { content } \\
(\%)\end{array}$ & $\begin{array}{c}\text { Grain } \\
\text { yield per } \\
\text { plant } \\
(\text { gm) }\end{array}$ \\
\hline \multicolumn{14}{|c|}{ Mean sum of squares } \\
\hline Replications & 2 & 0.000 & 0.001 & 0.005 & 0.004 & 0.000 & 0.005 & 0.926 & 1.688 & 0.001 & 0.064 & 0.152 & 1.088 \\
\hline Treatments & 35 & $0.033 * *$ & $0.038 * *$ & $0.381 * *$ & $0.142 * *$ & $0.010 * *$ & $0.711 * *$ & $2338.327 * *$ & $\begin{array}{c}1162.144 \\
* *\end{array}$ & $4.306 * *$ & $32.493 * *$ & $86.673 * *$ & $2.883 * *$ \\
\hline Error & 70 & 0.001 & 0.002 & 0.027 & 0.012 & 0.001 & 0.024 & 48.069 & 7.994 & 0.031 & 0.480 & 2.543 & 1.299 \\
\hline Total & 107 & 0.035 & 0.040 & 0.413 & 0.158 & 0.012 & 0.740 & 2387.321 & 1171.825 & 4.338 & 33.036 & 89.367 & 5.269 \\
\hline
\end{tabular}


Table.3 Estimates of variability, heritability and genetic advance as per cent of mean for grain yield, yield component characters and quality trait of $28 \mathrm{~F}_{1} \mathrm{~S}$ and 8 parents in rice (Oryza sativa L.)

\begin{tabular}{|c|c|c|c|c|c|c|c|c|c|}
\hline \multirow{2}{*}{$\begin{array}{l}\text { S. } \\
\text { No. }\end{array}$} & \multirow[t]{2}{*}{ Characters } & \multirow[t]{2}{*}{ Mean } & \multicolumn{2}{|c|}{ Range } & \multicolumn{2}{|c|}{ Coefficient of variation } & \multirow{2}{*}{$\begin{array}{l}\text { Heritability } \\
\text { (broad sense) }\end{array}$} & \multirow{2}{*}{$\begin{array}{c}\text { Genetic } \\
\text { Advancement }\end{array}$} & \multirow{2}{*}{$\begin{array}{c}\text { Genetic advance } \\
\text { as per cent of } \\
\text { mean }\end{array}$} \\
\hline & & & Min. & Max. & $\operatorname{PCV}(\%)$ & GCV $(\%)$ & & & \\
\hline 1 & Days to $50 \%$ flowering & 94.35 & 68.67 & 108.00 & 8.96 & 8.73 & 94.80 & 16.51 & 17.50 \\
\hline 2 & Days to maturity & 123.69 & 97.67 & 138.00 & 6.88 & 6.63 & 92.80 & 16.28 & 13.16 \\
\hline 3 & Plant height (cm) & 114.90 & 92.90 & 130.18 & 7.56 & 7.35 & 94.50 & 16.91 & 14.72 \\
\hline 4 & Total number of tillers plant ${ }^{-1}$ & 14.87 & 10.33 & 17.67 & 14.29 & 12.73 & 79.40 & 3.47 & 23.36 \\
\hline 5 & $\begin{array}{l}\text { Number of ear bearing tillers } \\
\text { plant }^{-1}\end{array}$ & 11.85 & 8.67 & 14.00 & 12.75 & 9.38 & 54.10 & 1.68 & 14.21 \\
\hline 6 & Panicle length per plant $(\mathrm{cm})$ & 28.73 & 24.47 & 31.20 & 6.66 & 4.99 & 56.10 & 2.21 & 7.69 \\
\hline 7 & Number of grains per panicle & 213.78 & 141.23 & 270.89 & 13.74 & 12.69 & 85.20 & 51.59 & 24.13 \\
\hline 8 & Test weight (g) & 21.15 & 18.20 & 24.33 & 7.72 & 7.44 & 92.90 & 3.13 & 14.77 \\
\hline 9 & LAI at max tillering stage & 2.70 & 2.09 & 3.72 & 17.49 & 16.85 & 92.80 & 0.90 & 33.45 \\
\hline 10 & Grain yield plant $^{-1}(\mathrm{~g})$ & 28.75 & 20.21 & 38.03 & 19.24 & 18.42 & 91.70 & 10.45 & 36.33 \\
\hline 11 & Hulling (\%) & 79.85 & 77.82 & 82.24 & 1.69 & 0.91 & 28.90 & 0.81 & 1.01 \\
\hline 12 & Milling (\%) & 72.65 & 67.20 & 77.92 & 3.47 & 3.05 & 77.20 & 4.01 & 5.52 \\
\hline 13 & Head rice recovery $(\%)$ & 67.09 & 62.67 & 74.98 & 4.59 & 4.12 & 80.70 & 5.12 & 7.63 \\
\hline 14 & Kernel length (mm) & 5.67 & 5.31 & 6.06 & 2.99 & 2.61 & 76.20 & 0.27 & 4.69 \\
\hline 15 & Kernel breadth (mm) & 2.23 & 1.93 & 2.46 & 4.89 & 4.62 & 89.40 & 0.20 & 9.01 \\
\hline 16 & Length/Breadth Ratio & 2.54 & 2.37 & 2.90 & 4.62 & 4.30 & 86.60 & 0.21 & 8.24 \\
\hline 17 & Kernel length after cooking (mm) & 9.53 & 8.73 & 10.09 & 4.00 & 3.60 & 81.10 & 0.64 & 6.68 \\
\hline 18 & $\begin{array}{l}\text { Kernel breadth after cooking } \\
(\mathrm{mm})\end{array}$ & 2.89 & 2.56 & 3.25 & 8.14 & 7.20 & 78.20 & 0.38 & 13.12 \\
\hline 19 & Kernel Elongation Ratio & 1.68 & 1.55 & 1.77 & 3.92 & 3.19 & 66.40 & 0.09 & 5.35 \\
\hline 20 & Volume expansion ratio & 4.73 & 3.79 & 5.82 & 10.63 & 10.12 & 90.60 & 0.94 & 19.83 \\
\hline 21 & Water uptake (ml) & 183.29 & 121.67 & 256.67 & 15.54 & 15.08 & 94.10 & 55.21 & 30.12 \\
\hline 22 & Gel consistency (mm) & 67.69 & 41.66 & 98.27 & 29.28 & 28.98 & 98.00 & 39.99 & 59.08 \\
\hline 23 & Alkali spreading value (mm) & 4.30 & 2.50 & 6.17 & 28.08 & 27.77 & 97.90 & 2.43 & 56.60 \\
\hline 24 & Amylose content (\%) & 23.70 & 18.86 & 32.53 & 14.09 & 13.78 & 95.70 & 6.58 & 27.78 \\
\hline
\end{tabular}


The phenotypic coefficient of variance (PCV) was slightly higher in magnitude than genotypic coefficient of variance (GCV) for all the characters studied indicating the influence of environment on expression of these traits. Gel consistency and alkali spreading value showed high PCV and GCV. While, moderate PCV and moderate GCV was observed for total number of tillers per plant, number of grains per panicle, leaf area index at maximum tillering stage, volume expansion ratio, water upatake, amylase content and grain yield per plant, whereas number of ear bearing tillers per plant exhibited moderate PCV and low GCV. Plant height recorded moderate $\mathrm{PCV}$ and low GCV. However, low PCV, GCV were recorded for days to $50 \%$ flowering, days to maturity, plant height, panicle length, test weight, hulling per cent, milling per cent, head rice recovery, kernel length, kernel breadth, L/B ratio, kernel length after cooking, kernel breadth after cooking and kernel elongation ratio (Table 3). Similar results were reported by Allam et al., (2015), Navin Kumar et al., (2015), Sameera et al., (2015), Shajedur Hossain et al., (2015), Vijay Kumar et al., (2015), Arpita et al., (2014) and Gokulakrishnan et al., (2014).

High heritability coupled with high genetic advance as per cent of mean was observed for the characters viz., total number of tillers per plant, panicle length, number of grains per panicle, leaf area index at maximum tillering stage, water uptake, gel consistency, alkali spreading value, amylase content and grain yield per plant, indicating that the heritability was due to additive gene effects, which may be exploited through breeding methods involving simple selection like pedigree method, mass selection, ear-to-row method etc. are to be followed to improve these traits. Whereas high heritability and moderate genetic advance as per cent of mean was recorded for days to $50 \%$ flowering, days to maturity, plant height, test weight, kernel breadth after cooking and volume expansion ratio. While, number of ear bearing tillers per plant exhibited moderate heritability, genetic advance as per cent of mean, reveals that heritability was due to additive gene effects and selection may be effective. Milling per cent, hulling per cent, kernel length, kernel breadth, L/B ratio, kernel length after cooking and kernel elongation ratio showed high heritability coupled with low genetic advance as per cent of mean, indicative of non-additive gene action and heritability was being exhibited due to favorable influence of environmental conditions rather than genotype and selection for this trait may not be rewarded. Whereas moderate heritability coupled with low genetic advance as per cent of mean was noticed for panicle length and low heritability coupled with low genetic advance as per cent of mean for hulling per cent. These findings are corroborated by Keya et al., (2015), Sameera et al., (2015), Arpita et al., (2014), Gokulakrishnan et al., (2014) and Swati Sharma et al., (2014).

High to moderate variability was observed for total number of tillers per plant, number of grains per panicle, LAI at maximum tillering stage, volume expansion ratio, water uptake, amylose content and grain yield per plant. High heritability coupled with high genetic advance as per cent of means were observed for total number of tillers per plant, number of grains per panicle, leaf area index at maximum tillering stage, water uptake, gel consistency, alkali spreading value, amylose content and grain yield per plant, revealed the role of additive gene action. This in turn suggests the feasibility of simple selection procedures for improving these traits.

\section{Acknowledgement}

I humbly thank the authorities of Acharya N.G. Ranga Agricultural University-Guntur, Andhra Pradesh and University Grants Commission New Delhi for providing financial assistance in the form of fellowship during my course of study.

\section{References}

Allam, C.R., Jaiswal, H.K., Qamar, A., Challa Venkateshwarlu and Reddy, Y.S. 2015. 
Variability, heritability and genetic advance studies in some indegenous genotypes of basmati rice (Oryza sativa L.). Electronic Journal of Plant Breeding. 6(2): 506-511.

Arpita Shrivastava., Mishra, D.K., Koutu G.K and Singh, S.K. 2014. Heritability and genetic advance estimation from parental lines of hybrid rice. International Journal of Scientific Research. 3(7): 11-13.

Burton* G.W. 1952. Quantitative inheritance in grass. Proceedings of $6^{\text {th }}$ International Grass Land Congress. 1: 277-283.

Central Rice Research Institute (CRRI) - Indian Council of Agricultural Research. Cuttack (Odisha). -VISION 2050. http://www.crri. nic.in/ebookcrrivision2050_final_16Jan1 3.pdf

Gokulakrishnan, J., Sunil Kumar, B and Prakash, M. 2014. Variability studies for some yield and quality traits in rice (Oryza sativa L.). Plant Archives 14 (1): 533-536.

Hanson, W.O. 1963. Heritability in Statistical Genetics and Plant Breeding, Hanson, W.O and Robinson, H.F. (eds.), 1982. National Academy of Science and National Research Council, Washington D. C. pp. 125-139.

Johnson, H.W., Robinson, H.F and Comstock, R.E. 1955. Estimates of genetic and environmental variability in soybean. Agronomy Journal. 47: 314-318.

Keya Debnath., Bimal Das., Susbhamoy Sikder and Sarkar, K.K. 2015. Assessment of genetic variability character association and path coefficient for yield and its components characters in rice. The Ecoscan. 9 (1\&2): 455-459.

Melissa, A., Fitzgerald Susan, R., Mc Couch and Robert d Hall. 2009. Not just a grain of rice: the quest for quality. Trends in Plant Science. 14(3): 1360-1385.

Navin Kumar., Brijesh Tiwari., Gabrial M Lal., Mishra, S.P., Alka Katiyar and Yogeshwer Khuntey. 2015. Evaluation of rice hybrids (Oryza sativa L.) for yield and its component characters. Indian Research Journal of Genetics and Biotechnology. 7 (1): 41-43.

Sameera, S.K., Prasanna Rajesh, A., Jayalakshmi, V., Nirmala, P.J and Sriniva, T. 2015.Genetic variability studies for yield and yield components in rice (Oryza sativa L.). Electronic Journal of Plant Breeding. 6 (1): 269-273.

Shajedur Hossain., Maksudul Haque M.D and Jaamilur Rahman. 2015. Genetic variability, correlation and pth coeffficient analysis of morphological traits in some extinct local aman rice (Oryza sativa L.). Journal of Rice Research. 4 (1): 1-6.

Swati Sharma., Sanjay Singh., Rajkumar Kuldeep and Beniwal, D.C. 2014. Genetic variability and heritability estimates of rice new plant types lines for various quantitative traits. Agriculture for Sustainable Development. 2 (2): 137-140.

Vijay Kumar., Navin Kumar and Suresh, B.G. 2015. Systematic evaluation of exotic rice germplasm for yield components characters and its grain yield. International Journal of Research Studies in Biosciences. 3 (3): 53-56.

\section{How to cite this article:}

Hari Ram Kumar Bandi, P.V. Satyanarayana, D. Ratna Babu, N. Chamundeswari, V. Srinivasa Rao and Krishnam Raju, S. 2018. Genetic Variability Estimates for Yield and Yield Components Traits and Quality Traits in Rice (Oryza sativa L.). Int.J.Curr.Microbiol.App.Sci. 7(05): 551-559. doi: https://doi.org/10.20546/ijcmas.2018.705.069 